Portland State University

PDXScholar

Chemistry Faculty Publications and

Presentations

Chemistry

$1-1-2016$

\title{
Fluorescein Tri-Aldehyde Promotes the Selective Detection of Homocysteine
}

\author{
Aabha Barve \\ Portland State University \\ Mark Lowry \\ Portland State University \\ Jorge Escobedo \\ Portland State University \\ Josephrajan Thainashmuthu \\ Portland State University \\ Robert Strongin \\ Portland State University
}

Follow this and additional works at: https://pdxscholar.library.pdx.edu/chem_fac

Part of the Analytical Chemistry Commons, Biochemistry, Biophysics, and Structural Biology Commons, Biotechnology Commons, and the Medicinal-Pharmaceutical Chemistry Commons Let us know how access to this document benefits you.

\section{Citation Details}

Barve, A., Lowry, M., Escobedo, J.O. et al. (2016). Fluorescein Tri-Aldehyde Promotes the Selective Detection of Homocysteine. Journal of Fluorescence 26: 731.

This Citation is brought to you for free and open access. It has been accepted for inclusion in Chemistry Faculty Publications and Presentations by an authorized administrator of PDXScholar. Please contact us if we can make this document more accessible: pdxscholar@pdx.edu. 


\section{Effects of surface charge on the anomalous light extinction from metallic nanoparticles}

Article in Optics Communications · July 2016

DOI: 10.1016/j.optcom.2016.02.065

CITATIONS

0

2 authors, including:

Pui T Leung

Portland State University

147 PUBLICATIONS 1,653 CITATIONS

SEE PROFILE
READS

46 


\title{
Effects of surface charge on the anomalous light extinction from metallic nanoparticles
}

\author{
Edin Sijercic ${ }^{\mathrm{a}}$, P.T. Leung ${ }^{\mathrm{b}, *}$ \\ a Department of Electrical and Computer Engineering, Portland State University, P.O. Box 751, Portland, OR 97207, USA \\ ${ }^{\mathrm{b}}$ Department of Physics, Portland State University, P.O. Box 751, Portland, OR 97207, USA
}

\section{A R T I C L E I N F O}

\section{Article history:}

Received 11 January 2016

Received in revised form

19 February 2016

Accepted 27 February 2016

Keywords:

Anomalous scattering

Surface charge

Metallic nanoparticles

\begin{abstract}
A B S T R A C T
The effects of extraneous surface charges on the anomalous extinction from metallic nanoparticles are studied via an application of the extended Mie theory by Bohren and Hunt. Due to the sensitivity of the higher multipolar resonance on the surface charges, it is found that quenching of the anomalous resonance can be observed with presence of only a modest amount of charges on these particles. The observed effects thus provide a rather sensitive mechanism for the monitoring of the neutrality of these nanoparticles using far field scattering approaches.
\end{abstract}

(c) 2016 Elsevier B.V. All rights reserved.

\section{Introduction}

Optical scattering from particles of sub-wavelength sizes is a well-established subject of fundamental importance in both the understanding of the propagation of light through these particulate media, and in the monitoring of these particles via optical means [1]. Within classical electromagnetic theory, such understanding has been provided by the century-old celebrated theory of Lorenz [2] and Mie [3], which is an exact solution of Maxwell's equations for spherical particles. Moreover, despite its long history of being studied as evidenced by the large amount of literature published, the Lorenz-Mie (LM) theory has kept on generating surprises and intriguing results up to the present time. Two of such examples discovered more recently are the anomalous scattering $[4]$ and the Fano resonances $[5]$ in the scattering/extinction cross sections of the theory [6].

In several recent studies, Tribelsky and coworkers [4,5] have discovered the possibility of an "inverted hierarchy" in the multipole contributions to the LM theory for plasmonic materials of low dissipation, leading to the invalidation of the Rayleigh approximation. In addition, Miroshnichenko has observed similar Rayleigh breakdown with particles of negative refracting medium [7]. Aside from this anomaly observed in the total cross sections, intriguing phenomenon also showed up in the angular cross sections due to interference between different scattered wave components and/or the incident wave. For example, sharp asymmetric resonance of the Fano type was obtained in the forward and

\footnotetext{
* Corresponding author.
}

backward scattering cross sections near the surface plasmon resonance frequency of a Drude sphere [5].

Moreover, most of these previous studies [4-6] were limited to a neutral sphere as within the applicability of the LM theory. On the other hand, it has been pointed out since the late 1970s that the presence of surface charge on these particles will potentially change the scattering results due to the modification of the boundary conditions in the original LM theory [8]. The motivation of such a consideration comes from the fact that many particulate systems found both in Nature and in the laboratory are not perfectly neutral. These include, for example, light scattering from intergalactic dusts, water droplets in thunderstorm clouds, ...etc., as well as many colloidal systems prepared in the laboratory [9]. Thus, such "charge induced" optical effects in particle scattering has been an active research area for over three decades, including some very recent works [10-13]. Aside from the well-known small blue-shifts on the scattering/absorption resonances of these particles due to the effective increase in free charge density from the presence of the extraneous charges, some of the latest works [1012] have focused on certain specific and intriguing aspects of the charged-particle scattering problem such as the effects due to the non-uniformity in surface charge distribution; as well as those from the interband vs intraband transitions of the surface electrons. For example, it has been found that when the surface charges are not uniformly distributed on the particle, the original extended Mie theory of Bohren and Hunt (BH, [8]) must be modified to adopt a new version of the surface current density which will lead to the incorporation of higher order modes even in the long-wavelength limit [10-11]. Furthermore, the introduction of interband transition for the surface electrons has lead to slightly 
smaller and red-shifted cross sections with additional resonance peak emerging [12]. In addition to homogeneous solid particles, charged metallic nanoshells have also been studied with intriguing effects observed on the coupled surface plasmon modes [13].

In general, it has been found that such charge-induced optical effects are rather small in most far-field scattering process except for particles of very small sizes and a large amount of surface extraneous charges (i.e. a high surface voltage). Furthermore, dielectric particles will have a better chance to reveal such effects than metallic particles can, due to their characteristic frequencies being much lower than those of metals (the plasmon frequencies of metal are often in the visible or UV range for metals). On the other hand, if one were to optically probe these charged particles via near field interactions by having a localized light source placed in the vicinity of the particle, than it is possible to observe stronger charge-optical effects since these effects are more sensitive to the higher multipole resonance of the particle which can be excited more efficiently with the near field of a localized light source $[13,14]$. Nevertheless, near field experiments are not as convenient as those using far field especially when one refers to remote monitoring of these charged particles.

It is hence the purpose of the present work to provide new possibilities of efficient optical monitoring of these charged metallic particles via anomalous scattering. We shall demonstrate in the following that such scattering is rather sensitive to the presence of extraneous charges on these particles, leading to realistic monitoring of these charges via far field scattering. We shall focus on the inverted hierarchy of the multipole resonances in the total extinction cross section of these particles. The physical mechanisms behind this sensitivity will be elaborated. Similar charge sensitivity on the anomalous scattering from dielectric (nonmetallic) particles has been reported recently [15], based on blueshifted magnetic scattering rather than on the inverted hierarchy as demonstrated in the present work.

\section{Theory}

Let us consider the scattering of light by a spherical particle of radius $a$ immersed in a medium with an index of refraction $n_{m}$. The extinction cross section is derived from the exact Mie solution [1]:

$\sigma_{e x t}=\sum_{l=1}^{\infty} \frac{2 \pi}{k^{2}}(2 l+1) \operatorname{Re}\left(a_{l}+b_{l}\right)$

where summation $l$ is over all partial multipoles, $k=n_{m} \omega / c$ and $a_{l}$, $b_{l}$ are electric and magnetic scattering coefficients, respectively. These scattering coefficients can be written as (here we follow the notations of [4]):

$a_{l}, b_{l}=\frac{F_{l}^{(a, b)}(q, \varepsilon)}{F_{l}^{(a, b)}(q, \varepsilon)+i G_{l}^{(a, b)}(q, \varepsilon)}$

where $F_{l}^{(a, b)}$ and $G_{l}^{(a, b)}$ are defined in terms of the Riccati Bessel $\left(\psi_{l}\right)$ and Neuman $\left(\xi_{l}\right)$ functions as given in [1], $\varepsilon=\varepsilon_{p}(\omega) / \varepsilon_{m}(\omega)$ is the ratio between the dielectric function of the particle and that of the host medium, and $q$ is the dimensionless size parameter $(q=k a)$. Here, $\varepsilon_{m}(\omega)$ is assumed to be purely real and positive, whereas $\varepsilon_{p}(\omega)$ is complex in general and given by $\varepsilon_{p}(\omega)=\varepsilon_{p}^{\prime}(\omega)+i \varepsilon_{p}^{\prime \prime}(\omega)$. In order to allow for the excitation of surface plasmons, we will assume $\varepsilon_{p}^{\prime}(\omega)$ to be negative. In addition, a necessary condition to observe anomalous scattering is with small $\varepsilon_{p}^{\prime \prime}(\omega)$. Thus we first consider the following idealized Drude model for the metal particle, assuming $\varepsilon_{p}^{\prime \prime}(\omega)=0$ : $\varepsilon(\omega)=1-\frac{\omega_{p}^{2}}{\omega^{2}}$

where $\omega_{p}$ is the plasma frequency of the metal. As the frequency is swept, there will be a value of dielectric constant $\varepsilon$ for which $G_{l}^{(a)}=0$. When this occurs, the $a_{l}$ coefficient in Eq. (2) will attain the maximum value of unity. For various l's, this corresponds to resonant frequency of a multipole. Under the conditions of weakly absorbing metal, hierarchy in the scattering cross section of these resonances can be inverted, as referred to anomalous resonance by Tribelsky et al in [4].

In the long-wavelength or small particle size limit, we can expand the $F_{l}$ and $G_{l}$ in power series for small $q$ as follows [4]:

$F_{l}^{a} \approx q^{2 l+1} \frac{l+1}{[(2 l+1) ! !]^{2}} m^{l}\left(m^{2}-1\right)$

$G_{l}^{a} \approx m^{l} \frac{l}{2 l+1}\left\{\frac{l+1}{l}+m^{2}-\frac{q^{2}}{2}\left(m^{2}-1\right)\left[\frac{m^{2}}{2 l+3}+\frac{l+1}{l(2 l-1)}\right]\right\}$

$F_{l}^{b} \approx-\frac{m q^{2}}{2 l+1} F_{l}^{a}$

$G_{l}^{b} \approx-m^{l+1}\left[1+\frac{1-m^{2}}{2(2 l+1)} q^{2}\right]$

where $m=\sqrt{\varepsilon_{p} / \varepsilon_{m}}$ is the relative index of refraction.

To study the effects of surface charge on the anomalous scattering of plasmonic particles, we apply the following modified Mie coefficients as first obtained by Bohren and Hunt [8] (in the notations of [16]):

$a_{l}=\frac{\psi_{l}^{\prime}(m q) \cdot \psi_{l}(q)-m \psi_{l}^{\prime}(q) \cdot \psi_{l}(m q)-i \tau \psi_{l}^{\prime}(m q) \cdot \psi_{l}^{\prime}(q)}{\psi_{l}^{\prime}(m q) \cdot \xi_{l}(q)-m \xi_{l}^{\prime}(q) \cdot \psi_{l}(m q)-i \tau \psi_{l}^{\prime}(m q) \cdot \xi_{l}^{\prime}(q)}$

$b_{l}=\frac{\psi_{l}^{\prime}(q) \cdot \psi_{l}(m q)-m \psi_{l}^{\prime}(m q) \cdot \psi_{l}(q)+i \tau \psi_{l}(m q) \cdot \psi_{l}(q)}{\xi_{l}^{\prime}(q) \cdot \psi_{l}(m q)-m \psi_{l}^{\prime}(m q) \cdot \xi_{l}(q)+i \tau \psi_{l}(m q) \cdot \xi_{l}(q)}$

$\tau=i q \frac{\omega_{s}^{2}}{\omega\left(\omega+i \gamma_{s}\right)}$

where $\gamma_{s}=k_{B} T / \hbar$ is the damping constant, and $\tau$ the charge parameter expressed in terms of the surface plasmon frequency of the extraneous charge given by $\omega_{s}^{2}=N e^{2} / m_{e} a^{3}$, with $N, e, m_{e}$ the total number, the charge, and the mass of the excess charge, respectively. Thus a surface potential can be defined as $V=N e / 4 \pi \varepsilon_{0} a$. The temperature $T$ is set to $300 \mathrm{~K}$.

In the form of Eq. (2), the results in (5) can be put in the following form:

$a_{l}=\frac{F_{l}^{(a)}(q, \varepsilon)+i \alpha_{l}^{(a)}(q, \varepsilon, \tau)}{\left[F_{l}^{(a)}(q, \varepsilon)-\beta_{l}^{(a)}(q, \varepsilon, \tau)\right]+i \cdot\left[G_{l}^{(a)}(q, \varepsilon)+\alpha_{l}^{(a)}(q, \varepsilon, \tau)\right]}$

$b_{l}=\frac{F_{l}^{(b)}(q, \varepsilon)+i \alpha_{l}^{(b)}(q, \varepsilon, \tau)}{\left[F_{l}^{(b)}(q, \varepsilon)+\beta_{l}^{(b)}(q, \varepsilon, \tau)\right]+i \cdot\left[G_{l}^{(b)}(q, \varepsilon)-\alpha_{l}^{(b)}(q, \varepsilon, \tau)\right]}$

where $\alpha$ and $\beta$ are correction terms to account for the surface charge and expansion to the lowest order of $q$ yields:

$\alpha_{l}^{(a)}(q, \varepsilon, \tau) \approx \tau \cdot q^{2 l} \frac{(l+1)^{2}}{[(2 l+1) ! !]^{2}} m^{l}\left(m^{2}-1\right)$

$\beta_{l}^{(a)}(q, \varepsilon, \tau) \approx \tau \frac{l \cdot(l+1)}{2 l+1} \frac{m^{l}}{q}$

$\beta_{l}^{(b)}(q, \varepsilon, \tau) \approx-\tau \frac{m^{l+1}}{2 l+1} q$

$\alpha_{l}^{(b)}(q, \varepsilon, \tau) \approx \tau \frac{q^{2 l+2} m^{l+1}}{[(2 l+1) ! !]^{2}}$

From Eqs. (4) and (7) we observe that 
$\alpha_{l}^{(a)} \propto q^{2 l}, \quad F_{l}^{a} \propto q^{2 l+1}, \beta_{l}^{(a)} \propto q^{-1}$ and $G_{l}^{a} \propto q^{0}$. Therefore, for small $\mathrm{q}$ and not too small $\tau$, we can neglect $F_{l}^{a}$ in the expression for $a_{l}$, as well $\alpha_{l}^{(a)}$ in the imaginary part of the denominator of $a_{l}$ for all values of $l$. Likewise, $\alpha_{l}^{(b)} \propto q^{2 l+2}, F_{l}^{b} \propto q^{2 l+3}, \beta_{l}^{(b)} \propto q^{2 l+2}$ and $G_{l}^{b} \propto q^{2}$. Under the same conditions, we can neglect $F_{l}^{b}$ in the expression for $b_{l}$, as well $\alpha_{l}^{(b)}$ in the imaginary part of the denominator of $b_{l}$ for all values of $l$. Imposing these conditions to keep to the lowest order in $q$, we can simplify Eq. (6) to yield:

$a_{l} \approx \frac{i \alpha_{l}^{(a)}(q, \varepsilon, \tau)}{-\beta_{l}^{(a)}(q, \varepsilon, \tau)+i \cdot G_{l}^{(a)}(q, \varepsilon)}$

$b_{l} \approx \frac{i \alpha_{l}^{(b)}(q, \varepsilon, \tau)}{\beta_{l}^{(b)}(q, \varepsilon, \tau)+i \cdot G_{l}^{(b)}(q, \varepsilon)}$

If we now apply the condition for resonance [4], $G_{l}^{(a)}=0$, the magnitudes of the scattering coefficient $a_{l}$ will behave as $q^{2 l+1}$, and for small $q$ tend to diminish. This thus provides a mechanism to quench the anomalous scattering since the diminishing of these modified coefficients will be more efficient for greater values of the multipole order, hence reversing the inverted hierarchy described above. This will be illustrated via numerical simulations in the next section.

\section{Numerical results}

In order to quantify the effects of the surface charge, we have computed the scattering cross section in Eq. (1) with the modified Mie coefficient in Eq. (6) for an ideal Drude particle in vacuum with a size parameter $q=0.2$. The plasma frequency of the metallic particle is assumed to be $3.77 \cdot 10^{15} \mathrm{rad} / \mathrm{s}$, a value close to that for aluminum which was proposed to be a likely candidate to manifest anomalous scattering [4].

We first consider a neutral sphere. Fig. 1 shows the results for the normalized extinction cross-section $\sigma_{\text {ext }} / \pi a^{2}$ (which is the same as the scattering cross section for zero damping) as a function of the normalized frequency $\omega / \omega_{s p}$, where $\omega_{s p}=\omega_{p} / \sqrt{3}$ is the frequency of the dipole resonance as $q \rightarrow 0$. The results show the inverted hierarchy consistent with [4] with the cross section magnitudes increasing for higher order multipoles. Note that higher order multipole resonances are very narrow (but finite in both the peak values and widths), due to the small size parameter $(q=0.2)$ and the absence of damping in the idealized Drude model in (3). The finite width for the dipole cross section arises mainly from what is known as radiative damping [17].
Next we repeat the calculation with a surface potential of $10 \mathrm{~V}$ applied to the particle and the results are shown in Fig. 2. From the capacitance of the sphere $C=4 \pi \varepsilon_{0} a$, where $a$ is the sphere radius (approximately $30 \mathrm{~nm}$ at $\omega=\omega_{s p}$ for $q=0.2$ ), we can estimate the number of free electrons for an applied potential of $10 \mathrm{~V}$ to be about 200 , which is consistent with the previously published results [11]. It is clearly seen that, in consistency with our above analysis, the anomalous inverted hierarchy disappears with the dipole resonance again dominates as expected in the Rayleigh limit, together with the almost complete quenching of the octupole contribution. Note that the very small blue shifts in the resonances [8-16] due to the presence of the surface charges are hardly noticeable with such a small surface potential applied to the particle.

The previous calculations were done for a relatively small size parameter $(q=0.2)$. As this parameter increases, the required voltage or surface charge to quench anomalous extinction will increase as well. In Fig. 3, we have plotted the minimum required voltages to quench either the quadrupole or octupole resonance as a function of the size parameter. To demonstrate the disappearance of the inverted hierarchy, the solid line shows the voltage at which the amplitude of the quadrupole resonance has decreased to become just equal to that of the dipole resonance; and the dashed line shows the required voltage to reduce the octupole resonant peak to the same amplitude as the quadrupole peak, respectively. As can be observed, only much lower voltages are required to suppress inverted hierarchy with octupole resonance, and this phenomenon can be generalized to multipole contributions of even higher orders.

All the calculations done so far assume the nondissipative limit, which is an idealization. To investigate the effects of damping, we replace Eq. (3) with the following more realistic Drude function: $\varepsilon(\omega)=1-\frac{\omega_{p}^{2}}{\omega\left(\omega+i_{\gamma}\right)}$, where $\gamma$ is damping constant. In Fig. 4 we have plotted the required damping, normalized to the plasma frequency $\omega_{p}$, for the suppression of the quadrupole resonant peak amplitude to equal to that of the dipole peak. Such "critical damping" for this neutral sphere is seen to have an almost exponential dependence on the size parameter.

To illustrate the "quenching effect" of the anomalous extinction for this more realistic sphere with damping, we have plotted in Fig. 5 the extinction cross-section for a particle with a size parameter $q=0.25$ and normalized damping $10^{-6}$. As this is below the critical damping shown in Fig. 4, inverted hierarchy emerges. However, with an application of a modest surface voltage of $35 \mathrm{~V}$ will restore the normal hierarchy as shown in Fig. 6.

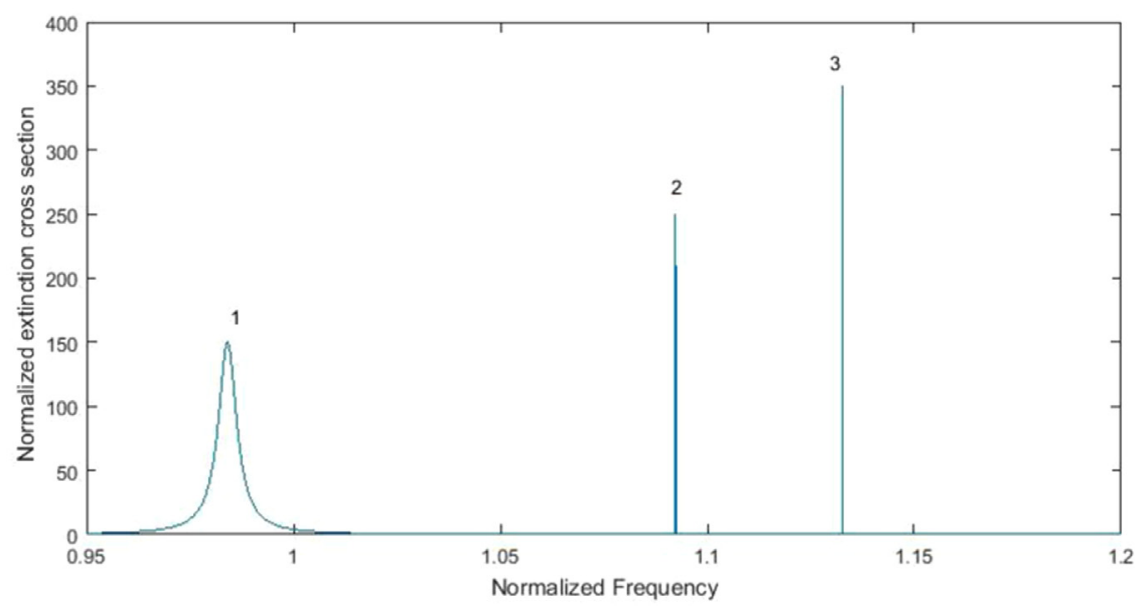

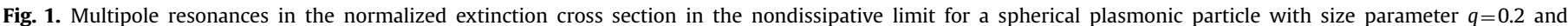
resonance peaks for: dipole (1), quadrupole (2) and octupole (3) resonances. 


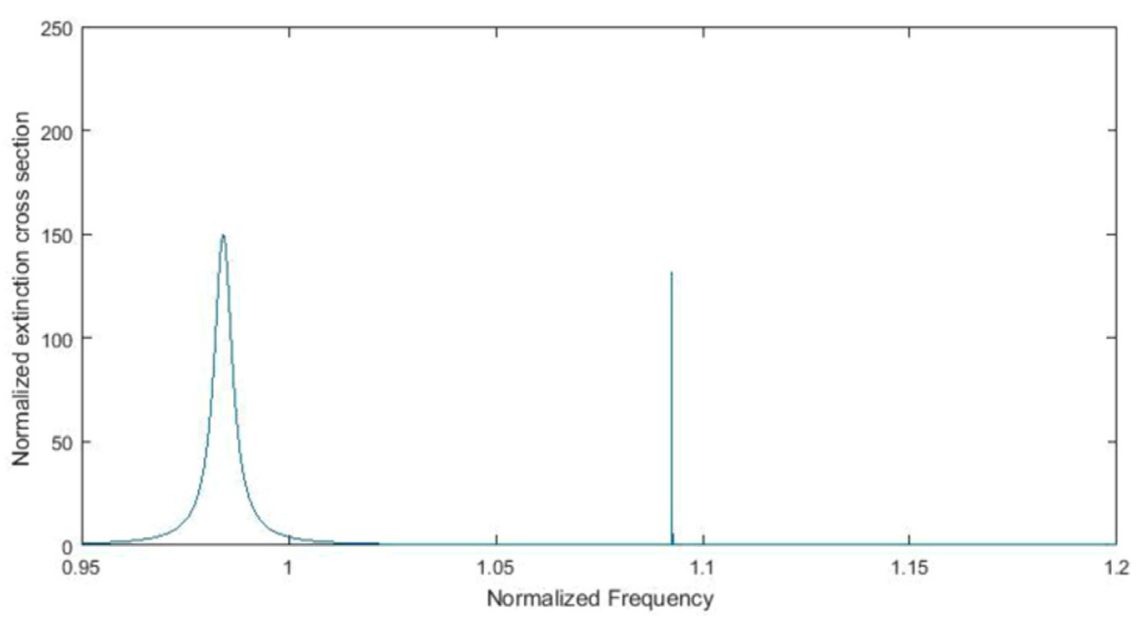

Fig. 2. As in Fig. 1, with a surface voltage of $10 \mathrm{~V}$ applied to the sphere.

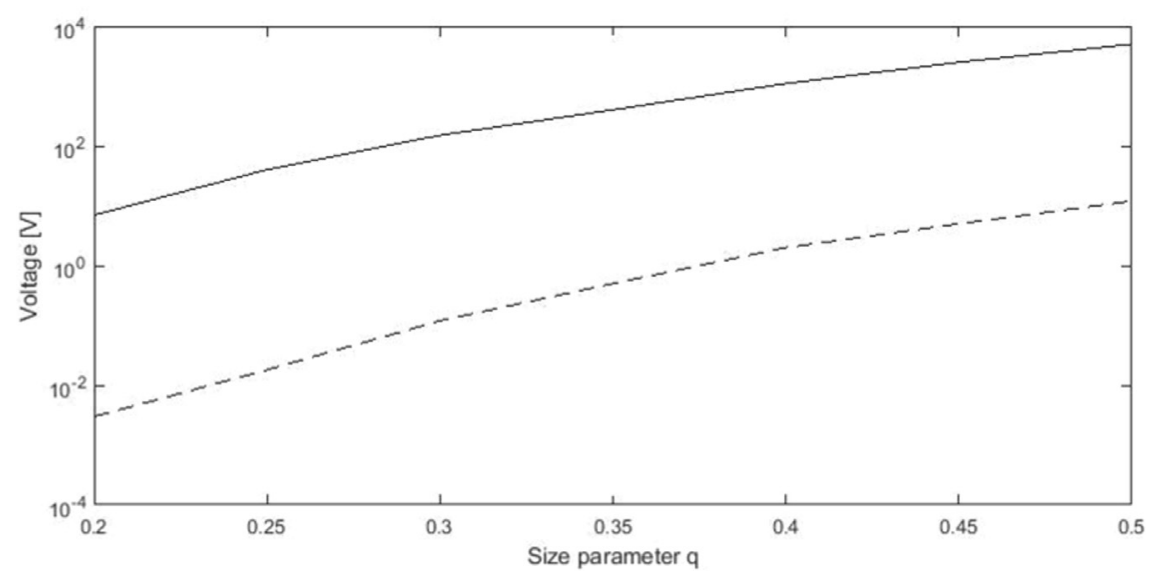

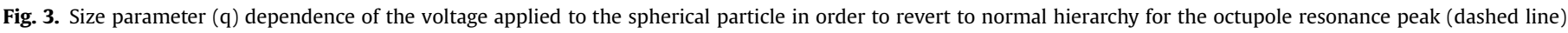
and quadrupole resonance peak (solid line) for the idealized plasmonic sphere.

\section{Discussion and conclusion}

In his paper we have demonstrated that adding surface charge on a small metallic (plasmonic) particle with low loss quenches anomalous extinction and reverts to normal hierarchy in multipole resonance peaks. The physical mechanism behind this quenching effect arises from the damping contributed by the surface conductivity associated with the extraneous charges, together with the fact that optically, such effects are more significant for the higher multipole scattering than for the lower one. Such damping will lead to the suppression and broadening of the scattering peak for various multipole resonance.

Moreover, this is completely insignificant for the dipole resonant peak due to the dominant effect of the radiative damping. We have also observed that this quenching effect is more appreciable for smaller particle size and for higher multipoles. This is due to the narrower resonances for the higher multipole resonances, and is consistent with previous observations for the

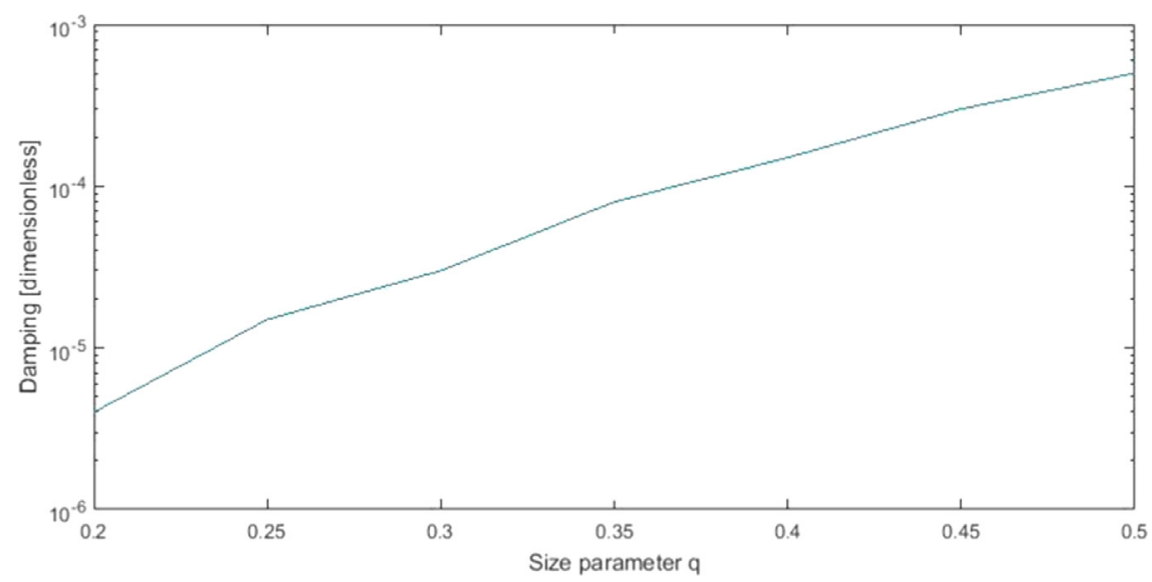

Fig. 4. Size parameter $(q)$ dependence of the required damping in the Drude model to revert to normal hierarchy for the quadrupole resonance peak. 


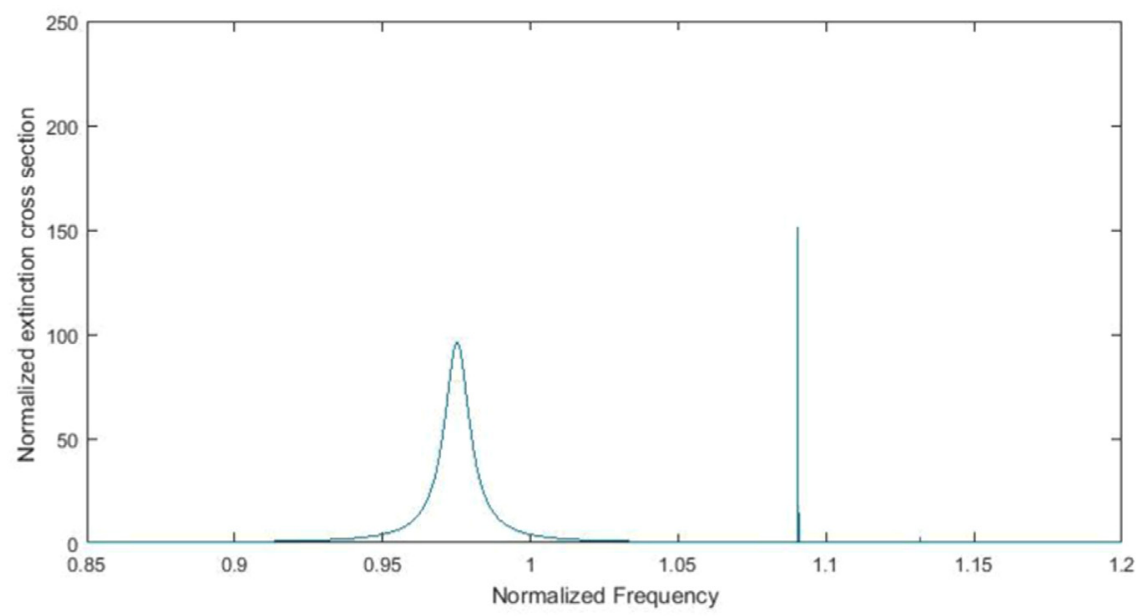

Fig. 5. Anomalous scattering in the normalized extinction cross section with a normalized damping of $1 \mathrm{e}-6$ for a spherical particle with size parameter $q=0.25$.

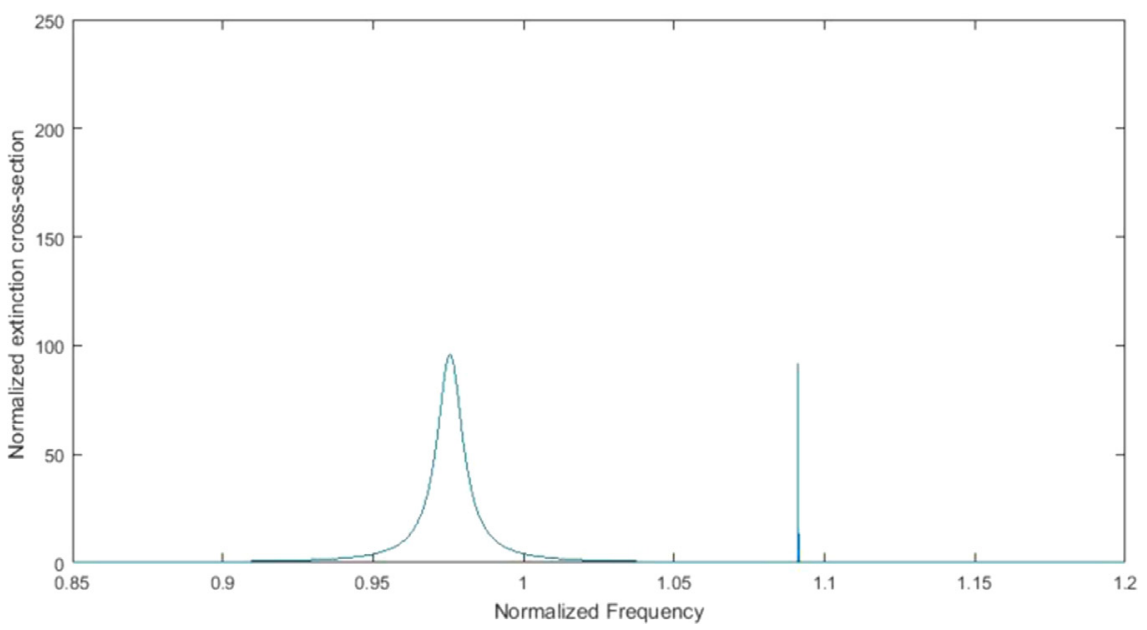

Fig. 6. Quenching of anomalous scattering for the sphere in Fig. 5 with an application of a surface voltage of 35 V.

significance of charge-optical effects for near fields $[13,14]$. Note that had we plotted the cross section for the scattering instead of the one for the extinction in our above analysis, the "critical" voltage (Fig. 3) and damping (Fig. 4) will become even smaller in values. We hence conclude that it may be possible to monitor the neutrality of small metallic particles of weak dissipation using farfield scattering via the recently-proposed anomalous scattering phenomenon reported in the literature [4-6]. Experimental confirmation of such possibility will be of significance in providing an efficient method for remote monitoring of the charging status of these particles.

\section{References}

[1] C.F. Bohren, D.R. Huffman, Absorption and Scattering of Light by Small Particles, Wiley, New York, 1983.

[2] L. Lorenz, Lysbevaegelsen i og uden for en af plane Lysbolger belyst Kugle, K. Dan. Vidensk. Selsk. Skr. 61 (1890) 1-62 (Række, 6).

[3] G. Mie, Contributions on the optics of turbid media, particularly colloidal metal solutions, Ann. Phys. 25 (1908) 377-445.

[4] M.I. Tribelsky, B.S. Luk'yanchuk, Anomalous light scattering by small particles, Phys. Rev. Letts. 97 (2006) 263902.

[5] M.I. Tribelsky, S. Flach, A.E. Miroshnichenko, A.V. Gorbach, Y.S. Kivshar, Light scattering by a finite obstacle and Fano resonances, Phys. Rev. Lett. 100 (2008) 043903.
[6] X. Fan, W. Zheng, D.J. Singh, Light scattering and surface plasmons on small spherical particles, Light: Sci. Appl. 3 (2014) e179, http://dx.doi.org/10.1038/ Isa.2014.60.

[7] A.E. Miroshnichenko, Non-Rayleigh limit of the Lorenz-Mie solution and suppression of scattering by spheres of negative refractive index, Phys. Rev. A $80(2009) 013808$

[8] C.F. Bohren, A.J. Hunt, Scattering of electromagnetic waves by a charged sphere, Can. J. Phys. 55 (1977) 1930-1935.

[9] J. Rostalski, M. Quinten, Effect of a surface charge on the halfwidth and peak position of cluster plasmons in colloidal metal particles, Colloid Polym. Sci. 274 (1996) 648-653.

[10] J. Klacka, M. Kocifaj, F. Kundracik, G. Videen, Optical signatures of electrically charged particles: fundamental problems and solutions, J. Quant. Spectrosc. Radiat. Transf. 164 (2015) 45-53.

[11] M. Kocifaj, J. Klacka, F. Kundracik, G. Videen, Charge-induced electromagnetic resonances in nanoparticles, Ann. Phys. 527 (2015) 765-769.

[12] J. Klacka, M. Kocifaj, F. Kundracik, G. Videen, I. Kohut, Generalization of electromagnetic scattering by charged grains through incorporation of interband and intraband effects, Opt. Lett. 40 (2015) 5070-5073.

[13] T. Bian, R. Chang, P.T. Leung, Optical interactions with a charged metallic nanoshell, J. Opt. Soc. Am. B 33 (2016) 17-26.

[14] M. Kocifaj, J. Klacka, Scattering of electromagnetic waves by charged spheres: near-field external intensity distribution, Opt. Lett. 37 (2012) 265-267.

[15] R.L. Heinisch, F.X. Bronold, H. Fehske, Optical signatures of the charge of a dielectric particle in a plasma, Phys. Rev. E 88 (2013) 023109.

[16] H.Y. Chung, P.T. Leung, D.P. Tsai, Effects of extraneous surface charges on the enhanced Raman scattering from metallic nanoparticles, J. Chem. Phys. 138 (2013) 224101

[17] M. Meier, A. Wokaun, Enhanced fields on large metal particles: dynamic depolarization, Opt. Lett. 8 (1983) 581-583. 